\title{
Crisis Communication on Twitter during a Global Crisis of Volkswagen - The Case of "Dieselgate"
}

\author{
Stefan Stieglitz, Milad Mirbabaie, Tobias Potthoff \\ University of Duisburg-Essen, Germany \\ \{stefan.stieglitz|milad.mirbabaie|tobias.potthoff\}@uni-due.de
}

\begin{abstract}
In this study, we investigate the communication behaviour in Twitter during the rise of a corporate crisis. In September 2015, the emission scandal of Volkswagen (also known as "Dieselgate") became public. We collected Twitter data and analysed approximately 400,000 tweets regarding the Volkswagen crisis. We take different perspectives on the data, by 1) separating the overall communication in peak and quiet phases, 2) analysing the sentiment in each phase, 3) looking at specific tweet contents, and 4) using statistical analyses to determine the significance of differences. Furthermore, we mapped the publishing behaviour of official Volkswagen accounts to the situational crisis communication theory (SCCT). The findings suggest that Volkswagen followed a strategy that is not covered by SCCT, i.e. keeping silent. Volkswagen's tweets were not able to reduce the emotionality and sentiment of the ongoing Twitter discussion. Instead, even during quiet phases, the communication remained rather negative.
\end{abstract}

\section{Introduction}

Social media has evolved into an important channel for enterprise online communication. Platforms such as Twitter, Facebook, and YouTube support low-cost advertisement, target-group-specific communication, and rapid dissemination of corporate content $[17,41]$. Additionally, social media platforms allow two-way communication between social media users (e.g. customers) and employees of a company $[33,41]$. As a result, massive amounts of data are generated, such as text, information about the author, and follower-followee relationships [37]. The analysis of online communication offers insights about how certain topics or brands are discussed in social media [17]. An ongoing monitoring of social media content might help to gather information about the perception of a company, a brand, or certain marketing campaigns [14]. Moreover, interaction with social media users might also help to increase the awareness and the reputation of a company $[21,44]$. However, in times of unanticipated issues that affect a product or entire organisations, social media analytics can help companies develop and adjust communication strategies for improving the company's image and diminishing the impact of a crisis situation [6]. For companies it is getting increasingly important to learn how to react on social media during crises, because it might affect their reputation and market position seriously and permanently [43]. Crises-related communication is often highly recognised and strongly discussed in social media, resulting in peaks, emotional expressions, and information sharing. While there is already some understanding of the general dynamics of social media crisis communication, there is little knowledge about how companies behave during a crisis and if they are able to affect the communication and users' attitudes [23, 35]. Based on the possibility to easily and quickly share a message with a potentially large audience, people frequently use online social media to communicate about crisis events [40, 44]. The emerging importance of social media and its utilisation is therefore affecting the way how companies need to behave during crises [34].

In this paper, we investigate the Twitter communication that was generated during the rise of the "Dieselgate", which was a scandal of global scope and Volkswagen was threatened by litigation in many countries. The global scope of this scandal represented a threat to VW's performance. This case exemplifies the more general question of how emotionally charged discussions can diffuse through microblogging during scandals that can carry substantive performance implications. Even though some case studies on crisis communication in social media exist [38], there is still little understanding about how companies react in such situations. Therefore, in this case study, we provide and analyse empirical data to understand corporate behaviour during a global crisis situation. Our study is guided by the following research questions (RQ):

RQ1a: How do Twitter users communicate in the rise of VW's "Dieselgate"? 
RQ1b: How can the Twitter communication behaviour of VW during a rising crisis be explained?

RQ2: What implications can companies draw for their communication strategy based on a better understanding of user activity, sentiments, and content generation on Twitter during a corporate crisis?

The paper is structured as follows: First, we describe the background of crisis communication and theories providing communication strategies. Then, we present our research approach, which is complemented by a case description, our data collection and analysis techniques. The next section comprises the results that form the basis for the succeeding discussion against related theories and cases. This section also proposes further areas of investigation from a methodological and practical point of view. The paper ends with concluding remarks and an outlook for further research.

\section{Background}

\subsection{Crisis communication in social media}

In contrast to traditional mass media, online social media are considered as platforms that allow users to create timely messages and interactive conversations [41]. People publish situationally relevant information in social media sites based on their personal perceptions, activities, and what they gather from other media sites [17]. This enables the public to get insights into the situation first-hand close to real-time $[17,36]$. Moreover, news and media agencies publish real-time updates and announcements on Twitter during crisis events. Public users collect or create critical information and share them with their network of followers and audience [33]. Tweets that are sent during crisis situations may facilitate the spreading of information and contribute to situational awareness $[12,22,24,44]$. However, user generated content might include false information [15] or rumours [26], which can have a negative impact on the situation. However, effective corporate response can weaken the secondary customer communication and lower its effect on product purchase intentions [1].

During crisis situations, the use of Twitter differs compared to ordinary times [17]. Research revealed that information sharing and broadcasting activities during crises happens more frequently compared to general Twitter activity [17, 19]. Communication plays a major role when it comes to dealing with crisis situations [9]. When a crisis hits an organisation, the crisis situation needs to be analysed by the crisis team in a way that the organisation's response provides rapid and instructing information to the public. Crises represent a constant hazard to the image of an organisation, especially crises that could have been avoided by the enterprise [43].

During tense or critical periods, the public perception plays a key role in crisis management. However, given the complexity and large amounts of data, it is not trivial to gather this information [10]. Some studies already applied sentiment analysis to gain a better understanding of the users' perceptions and opinion of certain issues or brands [32]. For example, [5] measured the overall emotionality in Facebook discussions during the 'Ash Crisis'. On both examined airlines' Facebook pages, the users' collective sentiments were quite positive, which was surprising because customers found themselves in difficult and acute situations. The authors explain this observation by the fact that the crisis trigger was not caused by the airlines but by an unpredictable, natural incident. Similarly, [38] examined users' sentiments during Toyota's recall separately within the different peaks and quiet stages they identified. The study revealed that sentiment polarisation in times of peak stages is much higher than in 'quiet stages'. [28] analysed in their research the connection between corporate posts and users' exhibited sentiments. Their work revealed that users exhibited a stronger positive affect towards the company except for the three days of the peak. Whilst we have gained knowledge on how corporates communicate during not preventable crisis, we have a limited knowledge on the strategy of the organisation in preventable crises. Thus, it is necessary to investigate the communication of an organisation during this type of crisis. Existing literature seems to suggest that an organisation's response strategies, especially apologies that quickly accept blame, seem to hold the potential to ameliorate some of the negative sentiments that emerge.

\subsection{Situational crisis communication theory}

From the perspective of an organisation, the most significant aim of crisis communication is to reestablish the image of the organisation and customer confidence [43]. In general, crisis communication strategies focus on actions by organisations in direct response to a crisis during the crisis but also offer guidance for post-crisis actions [45]. The situational crisis communication theory (SCCT) categorises different types of crises in order to develop effective crisis response strategies [7]. It has its central focus on how to manage organisational reputation during crises [8]. A significant number of research articles focus on legitimacy $[11,29]$, which is often used interchangeably with the phenomenon of reputation. However, legitimacy is "a generalized perception or assumption that the actions of an entity are desirable, 
proper, or appropriate within some socially constructed system of norms, values, beliefs, and definitions" and „resilient to particular events“ [39]. The authors [11] highlight in their article the differences between legitimacy and reputation, both considered as types of social evaluation. We follow the aforementioned article's view that reputation differs from legitimacy and, thus, we focus on reputational threats during crises. The reputational threat depends on the crisis type. Hence, crisis managers should choose crisis response strategies that demonstrate acceptance of responsibility for the crisis and acknowledge victims' concerns $[6,8]$. The SCCT differentiates three crisis clusters based on attributions of crisis responsibility: (1) victim, (2) accidental, and (3) preventable cluster. The victim cluster and accidental cluster evoke minimal attributions of crisis responsibility [6]. However, the preventable cluster has very strong attributions of crisis responsibility (human-error product harm and organisational misdeed) and the event is considered as intentional [6]. Crisis situations that have been caused by the company itself usually can be categorized as being preventable. Depending on the assigned cluster, the SCCT suggests the application of different response strategies to be especially reasonable, either (a) deny, (b) diminish or (c) rebuild crisis response strategies.

\section{Research design}

\subsection{Case description and data collection}

Volkswagen AG (VW) is one of the globally leading car manufacturers headquartered in Germany. In 2008, VW presented an engine that barely fulfilled $\mathrm{CO} 2$ requirements in Europe - but not the ones in the US. To prevent jeopardising the product launch on the American market, VW manipulated engine software to pretend lower $\mathrm{CO} 2$ emission during tests.

To evaluate the communication during the VW emission crisis, we focused on Twitter as a social communication network, because: (1) the number of participating users and tweets is high, (2) Twitter communication, in response to emerging issues, is fast and spontaneous (also due to mobile-based participation possibilities), (3) Twitter provides an application programming interface (API) which enables us to gather data at scale on specific issues, and (4) Twitter is characterised by a high topicality of content [3, 35]. When the VW emission scandal became public, we started to track various topicrelated keywords on Twitter, using a self-developed Java tool that crawls data through the Twitter SearchAPI. As a next step, we stored the data in a MySQL database. All tweets containing the keywords ' $\mathrm{VW}$ ' and 'Volkswagen' were collected. This includes tweets and mentions of the two main corporate Twitter accounts (@VW and @Volkswagen). The VW emission scandal was first mentioned in public media on 18 September 2015. We included tweets starting from 17 September 2015 in our analysis to have a comparison sample of what the conversions were like before the crisis started to attract attention in public media. We decided to focus on a six-week timespan, as we were especially interested in the rise of the crisis. Thus, tracking continued until 30 October 2015.

The six-week raw data were pre-processed and limited to English and German tweets. We checked the dataset manually for tweets not related to the crisis or potentially generated by bots. We identified bots by looking at those accounts with many tweets. Such bots published, for example, several (VW) car offerings and were excluded.

\subsection{Data analysis}

For analysing the Twitter communication of Volkswagen during a global crisis and, thus, to answer our research questions, we use a combination of different methods, which we describe in the following. We argue that these steps are necessary to analyse the communication thoroughly.

3.2.1. Peaks and quiet phases. We first analyse the collected data by determining time-related clusters and peaks of tweets in time. This allows us to study the dynamics in the crisis, i.e. rising or amplification of communication and the decline of interest and hence, less tweets. As other studies did before [38], we calculate the tweet-rate per minute and determine the average value based on calendar days for the observed period. To detect "louder" (high tweet-rate) and "quieter" phases (low tweet-rate) we compute the median absolute deviation (MAD) for each day as a measure of statistical dispersion [20]. The MAD is expected to be more robust against extreme outliers than the mean absolute deviation or the standard deviation [20].

With the MAD, we determine those days with higher tweet rates: peak $=($ tweet rate $>$ mean + MAD $)$. A series of days that are marked either as peaks or quiet stages (non-peaks), are defined as a phase. We define a peak as a field with a positive mean and MAD above a value of 10.6 tweets/min. Quiet stages are defined as those time periods where the mean and MAD is below 10.6 tweets $/ \mathrm{min}$.

3.2.2. Sentiment analysis. Twitter data is commonly studied with (automated) sentiment analyses, as they help to classify the sender's meanings and sentiments, 
e.g. positive or negative opinions. Researching the communication during a corporate crisis calls to determine whether tweets convey a positive opinion about the topic or brand, or join in booing the organisation. Thus, we conduct a sentiment analysis with the tool SentiStrength ${ }^{1}$. The software has been widely used for academic purposes and uses a lexical list of sentiment-related terms and rules to analyse the emotionality of a tweet [42]. SentiStrength reports strength values for the two attributes positive sentiment ( 1 to 5 ) and negative sentiment ( -1 to -5$)$. A combined measure is the polarity, which adds up both values (polarity $=\mathrm{p}+\mathrm{n}$ ). This measure has also been called scale [42]. It indicates the overall sentiment of a tweet and has a negative value if the negative sentiment score is higher than the positive sentiment score and vice versa. If both sentiment values are the same, the tweet does not have a polarity, i.e. it is neutral. According to [31], polarity can also be understood as binary polarity that disregards the strength of the polarity and just indicates a negative (1 ) or a positive polarity $(+1)$. Another useful measure is the emotionality or emotionality divergence, respectively. The latter has also been introduced by [31]. It calculates the span between the positive sentiment score and the negative sentiment score (emotionality $=|\mathrm{p}|+|\mathrm{n}|$ ). It expresses how pronounced the scores are and can serve as a proxy for the emotionality of a tweet.

3.2.3. Inferential statistics. To further study the communication data collected in the first six weeks of the VW emission scandal, an interferential statistical data analysis is conducted with IBM SPSS Statistics (Version 22). We perform an in-depth analysis of the differences between peak and quiet phases regarding the polarity and emotionality measures. Since the complete dataset is too large for even nonparametric analyses, we create disproportionate subsamples of 3,000 tweets for each phase of the identified peak and quiet phases (disproportionate stratification) [16]. The disproportionately stratified sample is reasoned with the large differences between quiet and peak phases regarding the number of tweets.

3.2.4. Users, retweets and Volkswagen's role. First, we identify the most active users during the peak phases, because they are expected to influence the crisis communication largely. Hence, we rank the Twitter profiles by the number of tweets they published in the peak phases. The top 20 profiles (10 in P1 and 10 in P2) with the highest tweet count are grouped into verified and unverified users. Thereby, we adopt Twitter's view on such accounts to be of greater public relevance. Furthermore, the top users' tweets are examined in terms of retweet count and tweet type (text, image or link). Second, all tweets in our dataset are ranked by retweet number. The 30 most retweeted tweets are examined regarding their tweet type (text, image, link) and content. In addition, the user profiles of all top 30 most retweeted tweets are also analysed such as the top users. Finally, we analyse the original tweets published by the observed VW Twitter accounts. We classify the tweets independently into the SCCT categories for further evaluation of VW's communication strategy. If not all agree on the same category, we discuss the specific tweets and find a consensus. The evaluation also includes a separate sentiment analysis of the corresponding@replies by users to evaluate whether VW's tweets have an impact on the tone of discussion.

\section{Findings}

\subsection{Overview and sentiment analysis}

The total number of posts between the dates 17-092015 and 30-10-2015 reaches 399,203 tweets created by 121,528 unique users that participated in the conversation. Even though the VW emission scandal became public on 18 September, we observe that the number of tweets per day abruptly rises after four days. The tweet volume reaches its climax almost one week afterwards, on 2015-09-23, at 44,400 tweets. This is four times larger than the tweet volume in the first couple of days. From that point on, the tweet volume per day decreases almost constantly.

The results reveal that the number of positive tweets is, on average, much lower $(38,127)$ than the amount of neutral $(136,346)$ and negative tweets $(224,730)$. Twitter users spread the highest number of negative tweets towards VW especially between 2015 09-22 and 2015-09-25.

We observe two peak phases, between 2015-09-21 and 2015-09-25, as well as on 2015-09-28. We also determine three quiet phases on 2015-09-17, as well as 2015-09-26 to 2015-09-27, and 2015-09-29 to 201509-30. Table 1 depicts how many tweets and unique users are found in each phase and illustrates the results of the sentiments for each phase. The statistical analysis comparing peak and quiet phases is visualised in Figure 1. We applied nonparametric procedures (Mann-Whitney $U$ Test) with random subsamples for each phase to test the difference in the measure polarity and emotionality for significance.

1 http://sentistrength.wlv.ac.uk, last access: 31 August 2017 
Table 1. Number of tweets, unique users, and the results of the sentiments for each phase

\begin{tabular}{|c|c|c|c|c|c|}
\hline Phase & Time Frame (Year: 2015) & $\Sigma$ Tweets & $\Sigma$ Unique Users & Polarity & Emotionality \\
\hline Quiet 1 (Q1) & $17-09-20-09$ & 23,096 & 3,693 & -0.38 & 3.08 \\
\hline Peak $1(\mathrm{P} 1)$ & $21-09-25-09$ & 153,739 & 12,523 & -0.95 & 3.38 \\
\hline Quiet 2 (Q2) & $26-09-27-09$ & 25,553 & 7,200 & -0.91 & 3.41 \\
\hline Peak 2 (P2) & $28-09$ & 15,985 & 10,190 & -0.95 & 3.30 \\
\hline Quiet 3 (Q3) & $29-09-30-09$ & 180,830 & 1,960 & -0.65 & 3.14 \\
\hline & Total & 399,203 & 35,566 & -0.77 & 3.26 \\
\hline
\end{tabular}
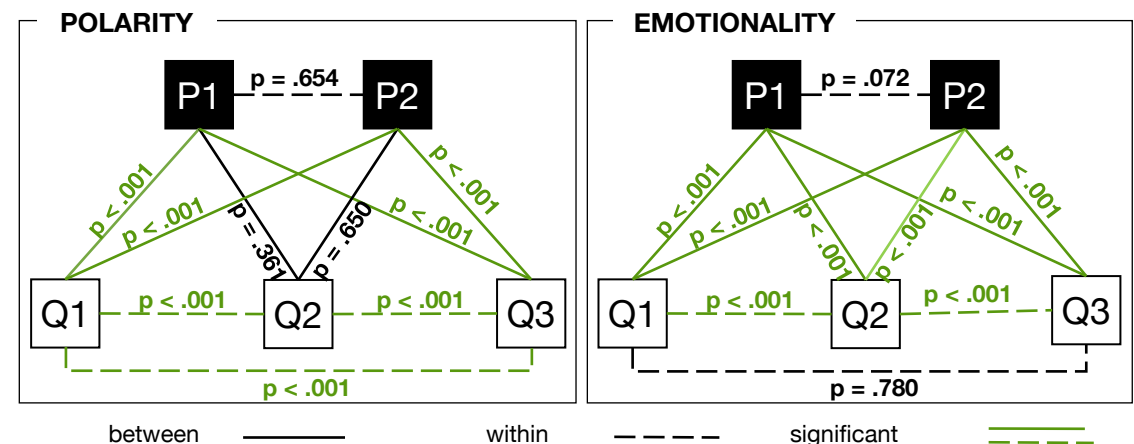

Figure 1. Polarity and emotionality differences between the peaks and the quiet stages

We found that the polarity of the peak phases is significantly more negative than the polarity of the quiet stages, $\mathrm{U}=24064671.50, \mathrm{z}=-11.77, \mathrm{p}<.001 . \mathrm{A}$ Kruskal-Wallis test reveals that there is a statistically significant difference in polarity between the different phases. Post-hoc tests were used to follow up on this finding. The results show that both peak phases are not different regarding the polarity $(U=-0.46, p=.65)$. Moreover, it appears that polarity is not different when P1 $(\mathrm{U}=-0.91, \mathrm{p}=.36)$ or $\mathrm{P} 2(\mathrm{U}=0.45, \mathrm{p}=.65)$ are compared to Q2. However, when Q1 is compared to P1 $(\mathrm{U}=13.65, \mathrm{p}<.001), \mathrm{Q} 2(\mathrm{U}=12.74, \mathrm{p}<.001), \mathrm{P} 2$ $(\mathrm{U}=13.19, \mathrm{p}<.001)$ and Q3 $(\mathrm{U}=4.75, \mathrm{p}<.001)$ polarity differences are significant. When Q3 is compared to Q2 $(\mathrm{U}=-7.99, \mathrm{p}<.001), \mathrm{P} 1(\mathrm{U}=-8.91$, $\mathrm{p}<.001)$ and $\mathrm{P} 2(\mathrm{U}=-8.45, \mathrm{p}<.001)$, it appears that polarity differences are significant.

The emotionality of the peak phases is significantly lower than those of the quiet stages: $\mathrm{U}=$ 25661692.50, $\mathrm{z}=-5,389, \mathrm{p}<.001$. A Kruskal-Wallis test reveals that there is a significant difference in emotionality between the different phases, $\mathrm{H}(4)=$ $304.47, \mathrm{p}<.001$. Post-hoc tests are used to follow up on this finding. It appears that emotionality is not different when $\mathrm{P} 1$ and $\mathrm{P} 2(\mathrm{U}=1.80, \mathrm{p}=.07)$ are compared, as well as when Q1 and Q3 $(\mathrm{U}=-0.28, \mathrm{p}=$ .78) are compared regarding emotionality. However, when Q2 is compared to P1 $(\mathrm{U}=-3.62, \mathrm{p}<.001), \mathrm{Q} 1$ $(\mathrm{U}=-12.14, \mathrm{p}<.001), \mathrm{P} 2(\mathrm{U}=5.42, \mathrm{p}<.001)$ and Q3 $(\mathrm{U}=11.86, \mathrm{p}<.001)$ emotionality differences are statistically significant. Furthermore, it appears that emotionality is different when P1 and Q1 $(U=-8.52$, $\mathrm{p}<.001$ ) are compared, as well as when Q1 and Q3 (U $=6.44, \mathrm{p}<.001)$ are compared regarding emotionality.

Studying the emotionality measure that "captures the extent of emotional expression" [31:545] in our sample revealed that it differs significantly between each phase. We assumed the quiet phases to entail lower emotionality, as it has been seen in other cases [38]. Instead, the emotionality is compared to P1 significantly higher in Q2. Combining this result with the steady negative polarity, we preclude the explanation approach that more positive sentiments have caused the higher emotionality. Consequently, in Q2 the remaining users have reinforced their negative opinion although no further details or facts about the crisis have been published. The emotionality analysis supports that, even when there is a decrease of tweets, the topic still matters to people who seem to stimulate each other even in quiet phases.

\subsection{Analysis of users}

We analysed the top 10 users in the communication to understand the behaviour of the most active users. The examination of the users revealed that, in P1, they published 2,375 tweets within 5 days, ranging from 617 to 151 per account. Most users published exclusively either original tweets or retweets, $96 \%$ of those include URLs. Additionally, their individual average retweet count per tweet ranged from 0 to 14.89. In P2, the communication pattern looks similar. Here, all users, except for two, published either only tweets or only retweets as well. Detection of URLs 
reveals that $91 \%$ of the published 391 tweets or retweets contain URLs. Like P1, the retweet count of each user amounts to 0 and there were 8.37 average retweets per tweet. Moreover, there are four users who belong to the top users group in both peaks. Of these, two are probably related or have the same admin since they retweeted each other frequently and often refer to the same user account. By taking a closer look at the account description, it can be stated that in both peaks the top users in terms of published content are private persons (e.g. FreeAverageJoe), partially car- or technology-related (e.g. Pinnacle Auto Appraisers), as well as unverified news accounts, e.g. "TodaysCarNews" or "AllTheNewsIsNow". It is noticeable that official media accounts like "nytimesbusiness" published fewer tweets than the unverified top users. Additionally, neither @VW nor (a) Volkswagen are part of the top users group since they jointly published only 10 tweets during the peaks.

To understand what type of content reached a broad audience, in terms of being retweeted by other users, we analysed the top 30 retweets in the communication. The analysis reveals that the two most popular tweets in terms of being retweeted are satirical comments related to the crisis. The most retweeted news entry is a news message. It is noticeable that none of the top 3 retweets includes a hashtag and that the publishers' follower count varies between 37 (second) and 182,000 (first).

The examination of the account descriptions reveals that one third of the top retweets were generated by individuals' accounts unrelated to companies or general news sharing. Respectively, two thirds originate from partially verified news or media accounts. The examination of the tweets reveals that most tweets written by private persons do not include URLs; rather, they contain text or multimedia content. Additionally, those are mostly personal opinions or commentaries of the case and are often characterised by humorous remarks. In contrast, the other two thirds of the top retweets consist of news headlines and the related link, published by official newspaper accounts or third-party news hubs which pass on external information. Again, no tweets published by @VW nor @ Volkswagen can be found in the top retweets. Nevertheless, a detailed examination of the issued news articles revealed that $20 \%$ derived from the company's official press releases. Furthermore, it is noteworthy that 3 out of the top 30 retweets in the observed period are unrelated to the crisis and address topics like the international car exhibition IAA, a sponsored rally, and the introduction of a new model on the American market.

To get more insights into the content, the most frequent words in all tweets, retweets, and @ replies were analysed by counting the frequencies of all words with the application Wordstat 6 . The analysis revealed that the account @Volkswagen generated 28 original tweets. The account @ VW sent an average of 9 (a)replies per day, and only on weekdays. We found that the@Volkswagen account spread more original tweets (28) than the @VW account (6). However, none of the accounts retweeted any content. By examining the370@replies, we could observe that (a) $\mathrm{VW}$ continued to reply to general service inquiries which were not related to the crisis.

\subsection{Situational crisis communication theory}

We found 34 original tweets from VW. We classified these tweets to the crisis response strategies proposed in SCCT. Generally, we could classify four of 34 tweets only. Two tweets contained an apology from VW. Hence, we assigned the strategy apology in group rebuild crisis response strategies [6]. The first apology was sent on 2015-09-22 and the second was sent two days later, on 2015-09-24. Both apology tweets fall within phase P1. The two apology tweets evoked202 answers or@replies.

Two other tweets contained the Hashtag \#wirsindVW (German for "we are VW"). These tweets refer to the secondary crisis response strategy of ingratiation [6]. It is notably that these two tweets evoked four@replies only. The remaining 30 tweets could not be assigned to any SCCT response strategy, but 13 of these tweets contained crisis related information about the VW emission scandal. We found 17 remaining tweets with non-crisis-related content. Table 2 shows the quantity of tweets for each response category and the count of direct user replies upon these tweets.

Table 2. Number of tweets and @ replies with corresponding response strategies

\begin{tabular}{|l|l|l|}
\hline & $\begin{array}{l}\text { \# of } \\
\text { tweets }\end{array}$ & $\begin{array}{l}\text { \# of @ replies } \\
\text { of the users }\end{array}$ \\
\hline \multicolumn{3}{|l|}{ SCCT crisis response strategy } \\
\hline Rebuild strategy & 2 & 202 \\
\hline Bolstering strategy & 2 & 4 \\
\hline No SCCT crisis response strategy \\
\hline Crisis Related tweets & 13 & 184 \\
\hline $\begin{array}{l}\text { Non-Crisis Related } \\
\text { tweets }\end{array}$ & 17 & 55 \\
\hline
\end{tabular}

\section{Discussion}

Even though the case went public on 2015-09-18, the true extent started to become clear a few days later, 
with the beginning of P1 in our dataset. The intensity of the crisis raised until Martin Winterkorn resigned as CEO on 2015-09-23. Here, we also identified the largest amplitude of the tweet volume. On the day of P2, it was said that not only VW but also Audi and Skoda engines (both brands owned by VW) may be affected. Moreover, it was announced that German prosecutors opened preliminary inquiry into Martin Winterkorn. Regarding RQ1a - we see, in general, that new information being published affects the number of users involved in the communication. Especially during the peak phases, the participation of people is high, with 12,523 (P1) and 10,190 (P2) unique users per day, respectively. Previous studies also identified that more users enter the discussion in peak phases and in the rise of the communication in Twitter [25, 38]. We observed that communication peaks do not occur because users increase their tweet rate (on average), but because more unique users enter a discussion. This indicates that news and real-life incidents trigger the need to communicate in social media platforms.

\subsection{Polarity and emotionality}

Besides the basic quantities, our sentiment analysis revealed that the communication about the VW scandal on Twitter is characterised by negative opinions. On average, all phases have a negative sentiment polarity. The accusation of an intentional manipulation grossly violates trust and could have been expected to cause major negative opinions [38]. However, we observed and statistically tested the differences between the quiet and peak phases. The polarity in $\mathrm{P} 1$ is significantly more negative than it was before in Q1. That comes as no real surprise because the case and more details became public. Noteworthy, we found a still negative polarity in Q2 without significant difference to both $\mathrm{P} 1$ and $\mathrm{P} 2$. Consequently, the communication about the emission scandal remained negative although the number of tweets dropped to almost the same level than in Q1. At the same time, the number of unique users in Q2 is much lower than in P1, but is still twice as high as in Q1. Comparing to the last phase, the polarity becomes less negative in Q3. Here, we see less unique users participating than in every other phase. Regarding the polarity of Twitter communication during a corporate crisis we, therefore, propose the hypothesis that the communication's polarity is more strongly affected by the number of unique users than the number of tweets.

\subsection{Content of tweets in the rise of a crisis}

Besides the sentiment of tweets, we can learn much about the crisis communication by studying who is tweeting which content and to what extent. We differentiate between tweets containing URLs as an indicator for 'gate watching', since this practice is the collaborative identification and forwarding of situationally relevant information [2]. Attention should also be paid to the difference between top users and top retweets [30]. Top users can represent outgoing Twitter communication, which refers to what content is produced and published. Moreover, the top retweets reflect peoples' needs and interests in terms of sharing behaviour, because the more retweets and likes a tweet gets, the wider it spreads. Among the top ten users, $96 \%$ of their tweets contain URLs in P1, respectively $91 \%$ in P2. Looking at the most-retweeted tweets reveals that three quarters contain URLs. This information sharing behaviour implies that specific information is needed (or at least people feel like this is the case) and that they need to share this information, in the given example mainly in the form of news articles. As mentioned above, we excluded accounts with bot-like behaviour, i.e. high tweet-rate, only URLs without further text etc. In summary, it is noteworthy that $25 \%$ of the top retweets do not contain URLs, but are rather personal, often satirical, commentaries on the incident made by unverified accounts. This, in turn, can be characterised as 'audiencing' [13], since Twitter serves as a backchannel, meaning an unofficial and informational communication channel, where people talk back at latest events. The share of URLs is not as low as in the mainstream media events category of [4], but still indicates that there is not only a news seeking and sharing behaviour but also the need to get and share opinions - and to reveal one's own point of view by retweeting and liking corresponding tweets.

\subsection{The (missing) response of Volkswagen}

Addressing RQ1b and considering the total of 34 original tweets in the studied six-week period, of which only 17 tweets were crisis-related, we cannot testify a leading role as a communicator. VW did not make much use of the @,reply function to directly address stakeholders and customers. Neither did the company's tweets gain much attention or popularity. Additionally, the observation of both VW accounts reveals that they ignored tweets that were crisis-related but answered tweets that were mostly unrelated to the crisis, e.g. general customer service inquiries. VW received many inquiries and comments concerning the emission scandal but did not react to those tweets directly.

VW sent two tweets that could be classified as apology according to the SCCT. Such tweets are expected to have positive effects on people or their 
sentiment, respectively. In other words, the corporate apology should cushion the blow and catch prevailing negative opinions. This has been observed in previous studies, e.g. by [18]. Au contraire, in our case the @replies on the two apology tweets were more negative - even though not significantly. We see one reason for this different behaviour in the highly emotional engagement during the emission scandal, which affected many customers. Another explanation could be the timing of the apology; In the corporate crisis case of Domino's Pizza [18], an official apology video - which could weaken the sentiment negativity was published within 48 hours after the crisis went public. In our case, the crisis' trigger and scope may also account for the apology's ineffectiveness, since VW is assumed to have willingly deceived the global public and public administration over a long period.

Recalling our statistical analysis, the polarity and emotionality scores for the @replies reveal further insights. Messages directly addressed to official VW accounts were less negative than the general communication about the crisis. We see this difference based on users' perception of the official accounts as direct and genuine counterparts. Twitter users are more careful and moderate when writing to officials compared to sharing their opinion with their followers.

An evaluative summary of the role of VW and their actions reveals several challenges and opportunities the company faced on Twitter. Customer relationship management is a key factor of brand management and customer loyalty and, therefore, it is surprising that Volkswagen ignores crisis-related inquiries and focuses on daily business questions. It has to be considered that the management itself played a major role in causing this crisis. Therefore, the behaviour of ignoring the event could result in losing trust among customers and other stakeholders. We cannot say whether this inaction by Volkswagen is intentional. However, we propose that participation in the discussion is one of the key opportunities that occur. It is always a critical step to actively get in discourse with customers and the public, but since the VW accounts are perceived as reference accounts, a twoway exchange, which is one of the benefits of social media, must be included in a proper crisis communication. In this regard, addressing users' needs is another opportunity for VW. This can be done either through@replies or tweets that consider the overall interests and needs, which can be identified via word analysis, i.e. to detect co-occurrences and cluster the words to potential subtopics.

One factor, which is a challenge as well as an opportunity, is timely crisis communication. VW reacted rather quickly by publishing the video statement. However, they failed to explicitly mark it as an apology which may have had an influence on its perception and the likelihood to be watched.

\subsection{Crisis response strategies revisited}

In this last section, we want to discuss what we can learn from the VW case, thereby addressing RQ2.

Following previous case studies [26, 28, 38], it seems obvious that Twitter communication, in general, and crisis communication is shaped by periods of high and low concern in terms of public tweet volume, which corresponds to real live events and breaking news. Moreover, it is noticeable that public communication in social media is not necessarily negative but often affected by its trigger and SCCT cluster. Considering the few classified tweets of VW, we challenge whether the SCCT comprehensively depicts crisis communication strategies. In particular, we acknowledge that deliberately keeping silent may be a strategy in certain situations in which other strategies are expected to yield no success.

Though both strategies did not work as intended, this could have been also caused by the lack of conviction. Based on our analysis, which compared the polarity of @replies with the overall communication, we see more moderate tweets in @replies to the corporate accounts. Following, we propose the hypothesis that more@replies lead to a less negative discussion on Twitter and can be used by the organisations to calm down sentiments. Organisations can try to motivate and stimulate a discussion with more @replies to the corporate accounts, for example by asking questions.

However, it looks like the disclosed sentiments are most extreme in peak periods, so we assume that real live events and surprising news trigger people's need for speech and emotional engagement. Yet, our findings reveal that messages directly addressed to official accounts are less negative, which leads to the assumption that on Twitter, the corporate account is in fact perceived as a reference.

\section{Conclusion and outlook}

Conclusion. In this paper, we focus on the crisis communication of an enterprise and conduct a study on the Volkswagen scandal, which occurred in the year 2015. By examining the communication behaviour of the affected enterprise and participating users, the study reveals that the enterprise itself published way less original tweets than individuals or news and media accounts. Furthermore, the analysis of the top retweets reveals that they are personal, satirical commentaries on the incident. The sentiment analysis 
reveals that people engage emotionally even during the quiet stages.

Contributions. VW behave very passively in the crisis communication and did not respond to important users, such as opinion leaders. We argue that this behaviour has a negative impact on the communication and emotions associated with VW. Though we classified four tweets of VW as following a 'rebuild and bolstering' strategy per the SCCT model, VW provided very few crisis-relevant information or seemed to follow a strategy of keeping silent about the crisis, which is not covered in the SCCT model. At the same time, they continued to produce more non-crisis-related content, which cannot be deemed as an effective crisis management. Rather, it may have confused and upset participating users and potential customers. Further, the enterprise did not use Twitter's@ @replies feature for direct crisis-related communication and was not able to prevent significant negative emotions. We revealed in our study that organisations are seen as the main reference source in a crisis communication and, therefore, should make use of the benefits of social media, in terms of engaging actively with their users. In case of preventable cases according to the SCCT model, it can be stated that organisations should act more vividly and more transparently to avoid strong negative communication and a damage of their brand image and customer loyalty. Enterprises (in this case) may also make use of diminish crisis response strategies, or denying crisis response strategies [6]. Our paper also contributes to the literature on ways in which organisations communicate in social media, but also to literature focusing on and examining user behaviour during an organisational crisis.

Limitations. Our filter criteria reduced the data from more than 2 million tweets to less than 400,000 tweets. This step was necessary for the focus of our research to consider only topic-relevant tweets, but might have excluded potentially relevant content. Another limitation is the use of automated sentiment analysis. Although the results of the sentiment analysis can be used as a direction and describe the overall perception, in detail it does not always lead to accurate results. For example, the word 'scandal' was rated negative but it was often used to refer to the topic in general, as in 'VW scandal', rather than as a judgmental word. In addition, irony is often not interpreted correctly by the software. We did not consider in our sentiment analysis media content, such as images, videos or external links, but rather focused only on text.

Further Research. We suggest a validation of communication peaks based on sentiments and identification of practicability. Therefore, it would be relevant to identify potential differences, in terms of peak length as well as in terms of quantitative and qualitative research. Beyond the examination of the top retweets, a more detailed look into message dissemination would be necessary as well. Keeping silent as a strategy could be found in other cases as well, and SCCT would not predict such a strategy, but adopting a legitimacy-related lens, then "keeping silent" [27] underlines the motivation to spend more time on research that looks at the intersections of legitimacy and SCCT.

\section{Acknowledgment}

Many thanks are dedicated to our alumni Sina Youn, Philipp Wegge, Elisabeth Trubina, and Thomas Schafhauser for their support in analysing the data.

\section{References}

[1] Bi, G., B. Zheng, and H. Liu, "Secondary Crisis Communication on Social Media: the Role of Corporate Response and Social Influence in Product-Harm Crisis", PACIS 2014 Proceedings, (2014).

[2] Bruns, A., Gatewatching: Collaborative Online News Production, 2005.

[3] Bruns, A., and J. Burgess, "The use of Twitter hashtags in the formation of ad hoc publics", ECPR 2011, 1-9.

[4] Bruns, A., and S. Stieglitz, "Quantitative Approaches to Comparing Communication Patterns on Twitter", Journal of Technology in Human Services (30) (3-4), 2012, pp. 160185.

[5] Bygstad, B., and W. Presthus, "Social media as CRM? how two airline companies used facebook during the 'ash crisis' in 2010", SJIS, 25(1), 2013, pp. 51-71.

[6] Coombs, W.T., "Protecting organization reputations during a crisis: The development and application of situational crisis communication theory", Corporate Reputation Review 10 (3), 2007, pp. 163-176.

[7] Coombs, W.T., and S.J. Holladay, "Communication and Attributions in a Crisis: An Experimental Study in Crisis Communication", Journal of Public Relations Research 8 (4), 1996, pp. 279-295.

[8] Coombs, W.T., and S.J. Holladay, "Helping Crisis Managers Protect Reputational Assets: Initial Tests of the Situational Crisis Communication Theory", Management Communication Quarterly 16 (2), 2002, pp. 165-186.

[9] Coombs, W.T., and S.J. Holladay, The Handbook of Crisis Communication, 2010.

[10] Crawford, K., and M. Finn, "The limits of crisis data: analytical and ethical challenges of using social and mobile data to understand disasters", GeoJournal 80 (4), 2014, pp. 491-502.

[11] Deephouse, D., and M. Suchman, "Legitimacy in Organizational Institutionalism", The Sage handbook of Organizational Institutionalism(April), 2008, pp. 49-77.

[12] Ehnis, C., M. Mirbabaie, D. Bunker, and S. Stieglitz, "The role of social media network participants in extreme 
events", 25th ACIS, (2014).

[13] Fiske, J., "Audiencing: A cultural studies approach to watching television", Poetics 21(4), 1992, pp. 345-359.

[14] Gensler, S., F. Völckner, M. Egger, K. Fischbach, and D. Schoder, "Listen to Your Customers: Insights into Brand Image Using Online Consumer-Generated Product Reviews", International Journal of Electronic Commerce 20 (1), 2015, pp. 112-141.

[15] Gupta, A., H. Lamba, P. Kumaraguru, and A. Joshi, "Faking Sandy: characterizing and identifying fake images on Twitter during Hurricane Sandy", 22nd International Conference on $W W W, 2013$, pp. 729-736.

[16] Hansen, M.H., H. William N., and M. William G., Sample Survey Methods and Theory, Volume 1: Methods and Applications, 1993.

[17] Hughes, A.L., and L. Palen, "Twitter adoption and use in mass convergence and emergency events", International Journal of Emergency Management (6), 2009, pp. 248.

[18] Kim, H., J. Park, M. Cha, and J. Jeong, "The effect of bad news and CEO apology of corporate on user responses in social media", PLOS ONE 10(5), 2015, pp. 1-21.

[19] Kwak, H., C. Lee, H. Park, and S. Moon, "What is Twitter , a Social Network or a News Media?", IW3C2, 2010, pp. 1-10.

[20] Leys, C., C. Ley, O. Klein, P. Bernard, and L. Licata, "Detecting outliers: Do not use standard deviation around the mean, use absolute deviation around the median", Journal of Experimental Social Psychology 49 (4), 2013, pp. 764-766.

[21] Meske, C., and T. Potthoff, "The Dinu-Model - a process model for the design of nudges", ECIS 2017, 1-8.

[22] Mirbabaie, M., C. Ehnis, S. Stieglitz, and D. Bunker, "Communication roles in public events - A case study on Twitter communication”. IFIP 8.2, 2014, 207-218.

[23] Mirbabaie, M., S. Stieglitz, and M. Ruiz Eiro, "\#IronyOff - Understanding the Usage of Irony on Twitter during a Corporate Crisis.", Proceedings of the Pacific Asia Conference on Information Systems 2017, (2017).

[24] Mirbabaie, M., and E. Zapatka, "Sensemaking in Social Media Crisis Communication - A Case Study on the Brussels Bombings in 2016", 25th European Conference on Information Systems, (2017), 2169-2186.

[25] Mukkamala, R.R., J.I. Sorensen, A. Hussain, and R. Vatrapu, "Detecting Corporate Social Media Crises on Facebook Using Social Set Analysis", Proceedings - 2015 IEEE International Congress on Big Data, BigData Congress 2015, 2015, pp. 745-748.

[26] Oh, O., M. Agrawal, and H.R. Rao, "Community Intelligence and Social Media Services: A Rumor Theoretic Analysis of Tweets During Social Crises", MIS Quarterly 37 (2), 2013, pp. 407-426.

[27] Oliver, C., "Strategic Responses to Institutional Respones", The Academy of Management Review, 16, 1991, pp. 145-179.

[28] Park, J., M. Cha, H. Kim, and J. Jeong, "Managing Bad News in Social Media: A Case Study on Domino's Pizza Crisis.”, ICWSM, 2012, pp. 282-289.

[29] Patriotta, G., J.P. Gond, and F. Schultz, "Maintaining legitimacy: Controversies, orders of worth, and public justifications", Journal of Management Studies 48(8), 2011, pp. 1804-1836.
[30] Pervin, N., H. Takeda, and F. Toriumi, "Factors Affecting Retweetability: An Event-Centric Analysis on Twitter", ICIS 2014 Proceedings, (2014), 1-10.

[31] Pfitzner, R., A. Garas, and F. Schweitzer, "Emotional divergence influences information spreading in Twitter", The 6th International AAAI Conference on Weblogs and Social Media, 2012, pp. 2-5.

[32] Risius, M., and F. Akolk, "Differentiated sentiment analysis of corporate social media accounts", AMCIS 2015, pp. 1-13.

[33] Schultz, F., S. Utz, and A. Göritz, "Is the medium the message? Perceptions of and reactions to crisis communication via twitter, blogs and traditional media", Public Relations Review 37(1), 2011, pp. 20-27.

[34] Stephens, K.K., A.K. Barrett, and M.J. Mahometa, "Organizational communication in emergencies: Using multiple channels and sources to combat noise and capture attention", Human Communication Research 39 (2), 2013, pp. 230-251.

[35] Stieglitz, S., A. Bruns, and N. Krüger, "EnterpriseRelated Crisis Communication on Twitter", WI 2015, pp. 917-932.

[36] Stieglitz, S., D. Bunker, M. Mirbabaie, and C. Ehnis, "Sense-Making in Social Media During Extreme Events", Journal of Contingencies and Crisis Management (JCCM), 2017, pp. 1-17.

[37] Stieglitz, S., L. Dang-Xuan, A. Bruns, and C. Neuberger, "Social media analytics: An interdisciplinary approach and its implications for information systems", Bus. Inf. Sys. Eng., 6, 2014, pp. 89-96.

[38] Stieglitz, S., and N. Krüger, "Analysis of Sentiments in Corporate Twitter Communication - A Case Study on an Issue of Toyota", ACIS 2011 Proceedings, 2011.

[39] Suchman, M.C., "Managing Legitimacy : Strategic and Institutional Approaches, The Academy of Management Review, 20(3), 1995, pp. 571-610.

[40] Sutton, J., E.S. Spiro, B. Johnson, S. Fitzhugh, B. Gibson, and C.T. Butts, "Warning tweets: serial transmission of messages during the warning phase of a disaster event", Information, Communication \& Society 17 (6), 2014, pp. 765-787.

[41] Taylor, M., and D.C. Perry, "Diffusion of traditional and new media tactics in crisis communication", Public Relations Review 31(2), 2005, pp. 209-217.

[42] Thelwall, M., K. Buckley, G. Paltoglou, and D. Cai, "Sentiment Strength Detection in Short Informal Text", The American Society for Informational science and technology 61(12), 2010, pp. 2544-2558.

[43] Utz, S., F. Schultz, and S. Glocka, "Crisis communication online: How medium, crisis type and emotions affected public reactions in the Fukushima Daiichi nuclear disaster", Public Relations Review 39(1), 2013, pp. $40-46$.

[44] Vieweg, S.E., "Situational Awareness in Mass Emergency: A Behavioral and Linguistic Analysis of Microblogged Communications”, 2012, pp. 1-300.

[45] Yum, J.-Y., and S.-H. Jeong, "Examining the Public's Responses to Crisis Communication From the Perspective of Three Models of Attribution.", Journal of Business \& Technical Communication 29(2), 2015, pp. 159-183. 